\title{
Integrated care and diabetes: challenges, principles and opportunities
}

G D Tan

O Kozlowska

R D Rea

GD Tan DTM\&H FRCP DPhil is a Consultant Physician at the Oxford Centre for Diabetes, Endocrinology and Metabolism, Oxford University Hospitals NHS Foundation Trust, Oxford, and NIHR Oxford Biomedical Research Centre, UK. Competing interests: none declared.

O Kozlowska PhD is a Vice Chancellor's Research Fellow at Oxford Institute for Nursing and Allied Health Research, Oxford Brookes University, UK. Competing interests: none declared.

RD Rea DM FRCP is a Consultant Physician at Oxford Centre for Diabetes, Endocrinology and Metabolism, Oxford University Hospitals NHS Foundation Trust, Oxford, and NIHR-Oxford Biomedical Research Centre, UK. Competing interests: none declared

\section{Abstract}

The delivery of care for people with diabetes is a growing challenge with rapid growth in the numbers of people affected and increases in complexity of the management of the disease with more money being spent on diabetes than ever.

The system by which diabetes care is delivered in the UK is often fragmented and involves multiple providers across primary, community and specialist services which are managed by separate organisations, driven by different priorities, outcome measures and budgets, and supported by incompatible IT systems.

To improve the fragmentation of diabetes services, integrated care has been proposed as a solution. In essence, diabetes integration is the whole health community joining in partnership to own the health outcomes of patients with diabetes in their local area. This article examines what integration means to diabetes care, ranging from generalist to specialist diabetes care, describes the five key pillars of the integration of diabetes care and summarises key data sets which can be used.

\section{Keywords}

Commissioning of diabetes services; community care; diabetes care pathway; diabetes services; integrated care.

\section{Key points}

- Care of diabetes mellitus is often fragmented, with diabetes services delivered by different providers (including primary, intermediate and secondary care)

- Different providers often have different outcome measures based on historical organizational structures, rather than focusing on the patient as a whole

- Integrated care has been proposed as a solution to improve care for people with longterm conditions such as diabetes

- The key components of integrated care for diabetes are aligned financial incentives, integrated information technology, care planning, clinical engagement and partnership and robust shared clinical governance

\section{The challenge}

More people than ever have diabetes and the number of people experiencing complications or dying because of their diabetes is increasing. In the UK, someone is diagnosed with diabetes every 2 minutes; at least 10350 people have end stage renal failure due to their 
diabetes and more than 1700 people have their sight seriously affected by their diabetes every year. Every week, diabetes leads to 169 amputations, 680 strokes, 530 heart attacks and almost 2000 cases of heart failure in the UK. More than 500 people with diabetes die prematurely every week. The number of people diagnosed with diabetes has more than doubled over the past 20 years. We are living in a hidden epidemic of diabetes, which now affects 1 in 15 people in the UK.

Not only is there a significant clinical burden, diabetes and its complications lead to significant financial cost to health systems: in the UK, the NHS spends at least $£ 10$ billion a year on diabetes - about $10 \%$ of its total budget. Clinical developments in diabetes have led to an increasing number of new medications to treat diabetes, which cost considerably more than the drugs used to treat diabetes 20 years ago. In 2017-8, there were 53.4 million items prescribed for people with diabetes, an increase of $60 \%$ over only 10 years. Moreover, technology used to treat diabetes is rapidly advancing with the widespread introduction of flash glucose monitoring, continuous glucose monitoring, insulin pumps and closed loop systems for the treatment of type 1 diabetes.

The challenge to clinicians also inexorably increases. With the introduction of newer drugs to treat diabetes, combined with larger numbers of patients, clinicians in primary care can struggle to keep up with the latest advances as well as the increasing numbers of people with diabetes who need reviewing. Many practices have empowered practice nurses to deliver routine diabetes care, with the net result that primary care physicians feel deskilled when asked to deal with difficult clinical scenarios. In the meantime, treatments, their indications and contraindications become ever more complex.

Part of the increasing complexity derives from the fact that not only is the incidence of type 2 diabetes increasing, but the incidence and prevalence of type 1 and secondary diabetes also appear to be on the rise.

And it is not only clinicians who are becoming overloaded: over two thirds of people with diabetes don't fully understand their disease and over $40 \%$ of people with diabetes struggle with their psychological wellbeing. This may partly due to the high rates of multimorbidity in this group: over $85 \%$ of people with type 2 diabetes have one or more other comorbidity.

It against this backdrop of rising disease incidence and prevalence, increasing costs, ever more complex medications and technology, with increasing co-morbidity and a rising mental health burden that the systems of how diabetes care delivered has been questioned.

\section{The current situation}

The current UK model of diabetes care, with patients moving between primary, community and specialist care, does not always support clinicians' aims of delivering high quality, patient-centred care with the effective and efficient use of resources. Patients' complex needs may not be fully addressed, care is fragmented, and interventions may be delayed or duplicated.

Specific factors which may prompt a desire for change include:

- poor patient experience and poor patient outcomes with significant unwarranted variation in outcomes across geographical areas;

- lack of timeliness in the movement of patients between providers in a fragmented care system;

- frustration with the lack of communication of clinically relevant information between different clinical providers;

- unawareness of the most appropriate provider/location of care for a particular patient;

- a desire to share skills and knowledge between primary and secondary care to manage a whole population more effectively. 


\section{The concept of integrated care for diabetes}

Several national policies propose integration between primary and specialist care to improve the care of people with long-term conditions. NHS England promoted Integrated Care while professional associations for healthcare professionals have described how to dissolve traditional boundaries between general practice, community services, hospitals and social care.

Care can be integrated in a number of ways: integration between primary and secondary care, social and health care, physical and mental health. Whatever its form, integrated care is 'an organising principle for care delivery that aims to improve patient care and experience through improved coordination' with integration being 'a combined set of methods, processes and models that bring it about'. ${ }^{1}$

\section{Different ways to integrate care}

How integrated care may be organised spans from linkage, through coordination, to full integration with the latter recommended for care for people with severe, complex and longterm needs. ${ }^{1}$ Full integration does not necessarily mean organisations merging.

In diabetes, 'vertical integration' of care across traditional primary, community and secondary care providers is essential to reduce duplication of - and gaps in - services. This can be achieved by commissioning outcomes of whole pathways of care rather than fragments of a service.

\section{Components of an integrated diabetes service}

Figure 1 summarises the key components of integration in diabetes. ${ }^{2}$ In particular it highlights the need to have five essential pillars of integration in place in order to facilitate the provision of different elements of diabetes care. Integration of services around the patient and across the community becomes more robust and effective as more pillars are put in place.

\section{Integrated information technology (IT)}

A good information technology infrastructure is essential for the efficient running of a geographically disparate service. The system should:

- Allow timely communication between clinicians, both to seek advice and to communicate information

- Provide data to support population health, looking at quality of care across a geography

- Aid the clinical consultation rather than act just as a database. For example, if a patient has hypoglycaemic episodes ('hypos'), then the system should ask how severe they are; if the hypos are severe, does the patient drive? If they drive, then an information sheet should be able to be printed out to act as a focus of discussion around the legal issues of driving and hypoglycaemia

- Allow patients to access relevant information (results, care plans, appointment details)

\section{Aligned financial incentives and responsibilities}

This involves moving beyond a payment system which based on activity to a system which incentivises providers to deliver care centred around the patient. To achieve this, whole pathways of care need to be commissioned; components of diabetes care should not be commissioned individually because this leads to fragmentation of care and each provider fully using their budget with no incentive to save money to reinvest in other parts of the 
pathway. This efficient use and allocation of resources across the whole clinical pathway is key to delivering value-based healthcare with better outcomes and patient experience ${ }^{3}$.

At the time of writing this article, the tariff system which is used to identify, quantify and transfer money between commissioners and secondary care providers is under review. The tariff system is based on transactional payments and used in the NHS to pay for activity based care in a specialist setting and some community settings. Instead, the use of "blended payments" (which consist of a block contract with added incentives and a risk share agreement) is one proposal as to how to try to align financial and clinical responsibilities. However, whether such payment systems will allow whole pathways of care to be commissioned for people with diabetes, rather than just the "non-primary care" component, remains to be seen. Until "whole pathway" commissioning and financing can be introduced for people with diabetes underpinned by clinicians who have a responsibility for the same "whole pathway", then there will always be a barrier to integrated diabetes care.

\section{Care planning}

Care planning is a process that allows people with diabetes to have active involvement in deciding, agreeing and owning how their diabetes is managed. Care planning recognises that although healthcare professionals might have knowledge and expertise about diabetes in general, it's really only the person with the condition who knows how it impacts on their life. Both the person with diabetes and the healthcare team will then jointly agree the priorities or goals and the actions to take in response to this. Year of Care is an excellent example of implementation of a care planning process ${ }^{4}$.

\section{Clinical engagement and partnership}

All successful integrated models of care have involved clinicians and service users at an early stage. Local diabetes networks are in a unique position to work across natural diabetes communities which can bring together and facilitate a range of stakeholders from different disciplines with a mix of expertise, knowledge and competencies to deliver high-quality, cost effective care through the effective commissioning, organisation and delivery of services. In diabetes, networks exist in different formats and have different priorities, but should all have the principle of working in collaboration across a patient pathway to improve outcomes for people with diabetes. If networks do not exist there can be a lack of transparency of the diabetes service, poor communication and engagement between the pathway stakeholders especially with people with diabetes, the service is unlikely to be integrated and there is minimal opportunity to deliver improvement.

\section{Robust shared clinical governance}

Clinical governance in the context of integrated diabetes care is the whole diabetes healthcare community being responsible for the outcomes locally. Good communication, reporting and benchmarking will enable the provider organisations to review variation in outcomes and target resources as appropriate. This allows the whole diabetes community to both be responsible for the outcomes locally and have the financial ability to address the local priorities.

\section{Data and outcomes}

The local diabetes community should agree a variety of relevant outcomes such as those described in the "House of Outcomes" (Figure 2).

Diabetes is rich in data including nationally benchmarked data such as the National Diabetes Audit suite of audits 5 (Table 1) and data captured at GP level. The Improvement and Assessment Framework for clinical commissioning groups also contains metrics for diabetes around submission to the NDA, care processes and structured education. Such data 
can be used to drive down unwarranted variation and to improve care using methodology described by the Right Care programme ${ }^{6}$.

\section{Specific challenges}

The major challenge remains the alteration of a current mindset that is geared up to providing health services in silos. This requires a wholesale adoption of the five key elements of integration: shared governance, integrated IT, alignment of finances and responsibilities, care planning, clinical engagement and partnership with the aligning of agendas and incentives to focus on patient-centred care. Changes in policy making, regulation, financing and organisation of health care systems need to take place if meaningful outcomes are to be achieved.

\section{Glossary}

Commissioning of services: the continual process of planning, agreeing and monitoring services. This encompasses a range of actions from a health needs assessment of a population to deigning a clinical pathway to service specification and contract negotiation.

Tariff system: a set of prices and rules used by providers and commissioners of NHS care to deliver care to patients.

Year of Care: an approach to care and support planning providing a way fo working with patient-centred care and support for self management as central and core components. Local Diabetes Networks: a collaboration between a range of relevant stakeholders to provide the means to lead the planning, provision, monitoring and quality improvement of effective diabetes care at a local level. It provides the expertise and impetus for the development of quality services as well as the links between different organisations, perspectives and discplines to create a common vision.

Value based healthcare: the equitable, sustainable and transparent use of available resources and experiences for every person

\section{KEY REFERENCES}

1. Shaw S, Rosen R, Rumbold B. What is integrated care? Nuffield Trust, 2011. Available online at:

http://www.nuffieldtrust.org.uk/sites/files/nuffield/publication/what is integrated car e research report june11 0 .pdf last accessed 28/10/20

2. NHS Diabetes. Best practice for commissioning diabetes services. An integrated care framework. 2013. Available online at https://diabetes-resources-production.s3-eu-west1.amazonaws.com/diabetes-storage/migration/pdf/best-practice-commissioningdiabetes-services-integrated-care-framework-0313.pdf last accessed 28/10/20

3. Hurst L, Mahtani K, Pluddemann A, Lewis S, Harvey K, Briggs A, Boylan A-M, Bajwa R, Haire K, Entwistle A, Handa A and Heneghan C. Defining Value-based Healthcare in the NHS: CEBM report May 2019.

4. Year of Care https://www.yearofcare.co.uk/ last accessed 28/10/20

5. National Diabetes Audit https://digital.nhs.uk/data-and-information/clinical-audits-andregistries/national-diabetes-audit last accessed 28/10/20

6. Right Care https://www.england.nhs.uk/rightcare/ last accessed 28/10/20 


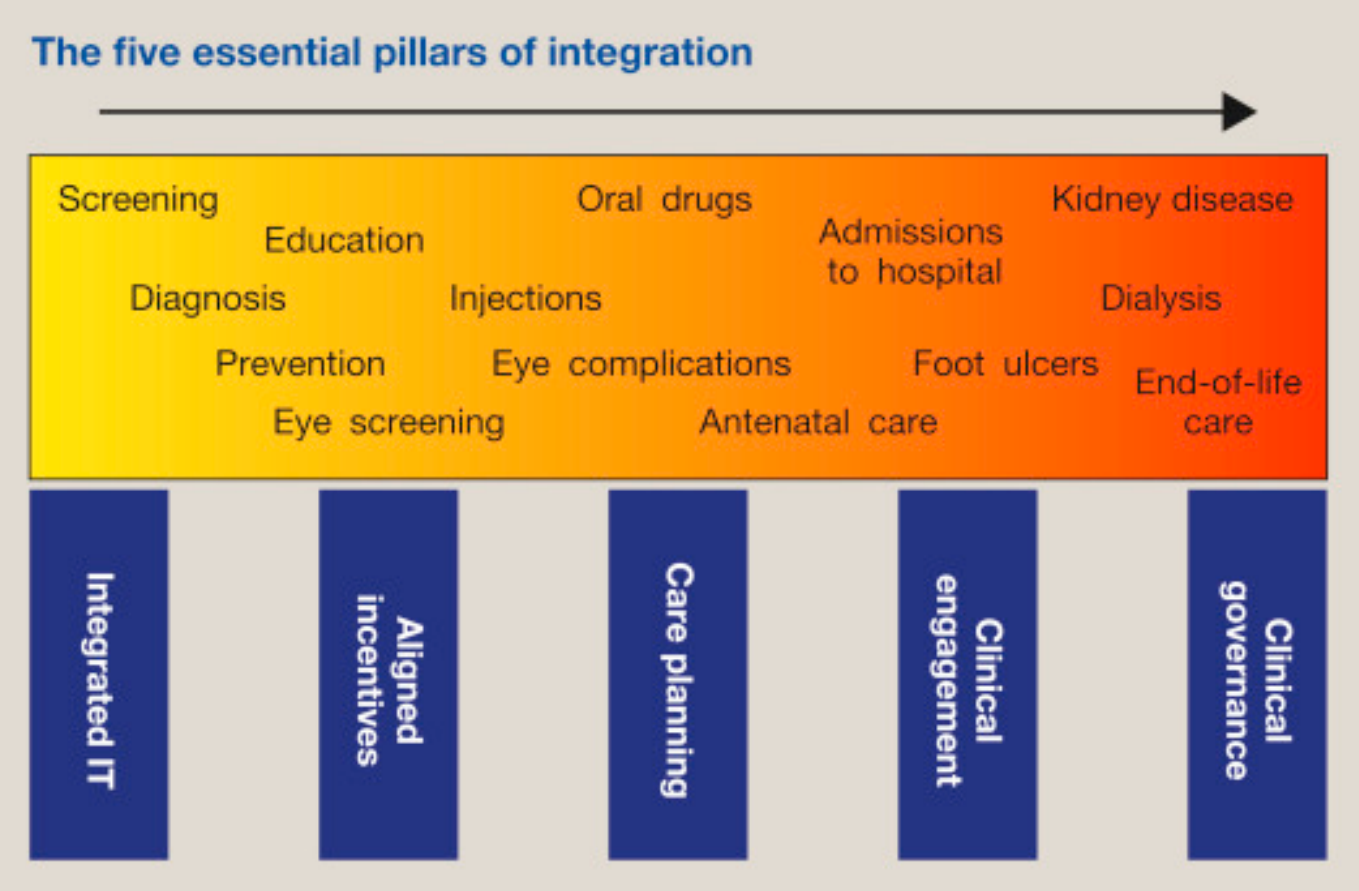

Figure 1 


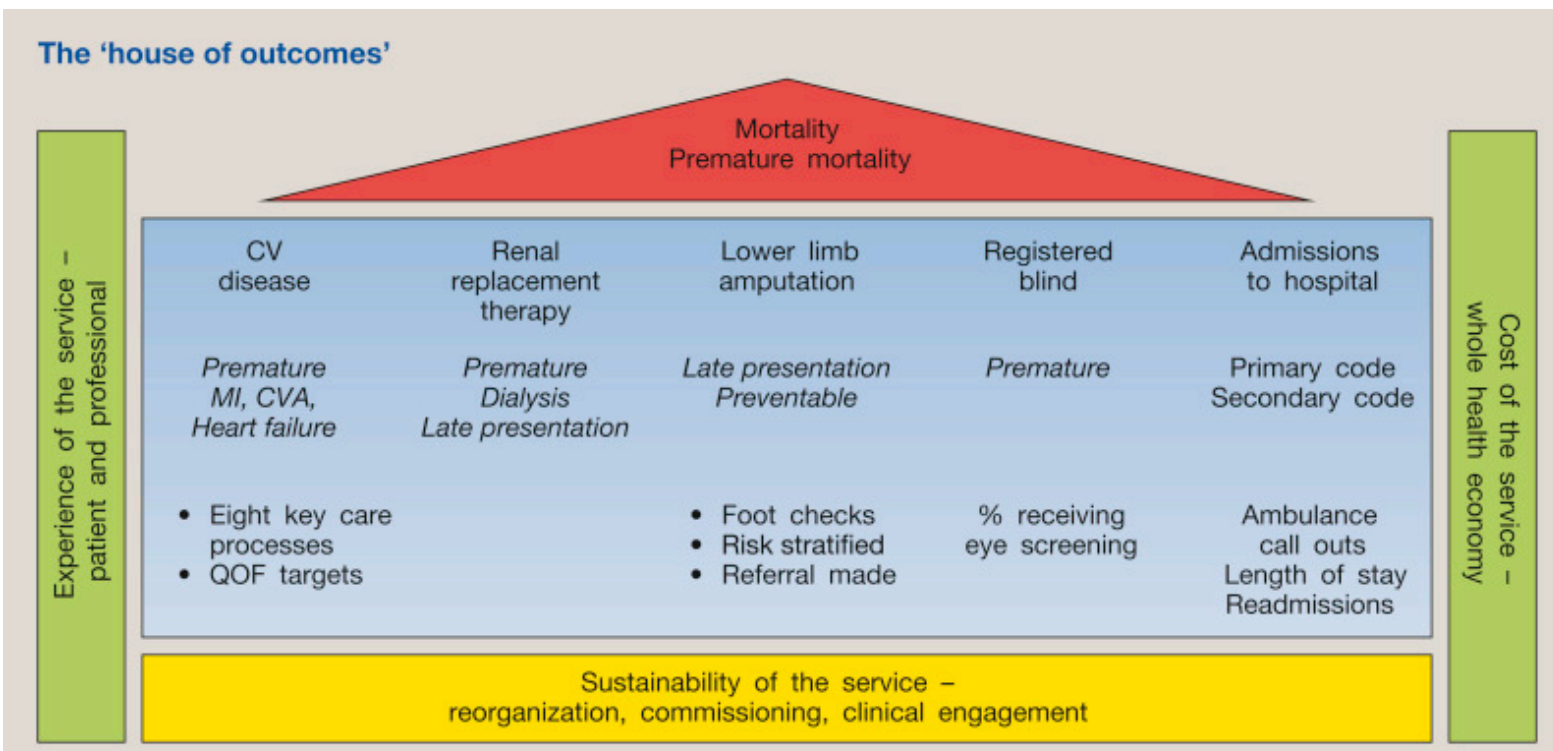

CVA, cerebrovascular accident; MI, myocardial infarction; QOF, Quality Outcomes Framework.

Figure 2 
The National Diabetes Audit suite

- Core diabetes audit (audits the care processes and outcomes of all people with diabetes treated in primary care and secondary care)

- National Diabetes in Pregnancy Audit (NPID)

- National Diabetes Foot Care Audit (NDFA)

- National Diabetes Inpatient Audit (NaDIA)

- Transition audit (a joint enterprise with the National Paediatric Diabetes Audit)

Table 1. 